\title{
A Report of 2 Cases of Disseminated Invasive Aspergillosis with Myocarditis in Immunocompromised Patients
}

\author{
Tetsuhiro Yoshino ${ }^{1,2}$, Hiroko Nishida ${ }^{1,3^{*}}$, Takashi Takita ${ }^{1}$, Masanori Nemoto ${ }^{1}$, Michihiro Sakauchi ${ }^{1}$, \\ Mami Hatano $^{4}$, Kazuyuki Koyama ${ }^{5}$, Masao Hori ${ }^{5}$, Katsuyuki Obara ${ }^{1}$ \\ ${ }^{1}$ Department of Internal Medicine, Mito Red Cross Hospital, Ibaraki, Japan; ${ }^{2}$ Center for Kampo Medicine, Keio University School of \\ Medicine, Tokyo, Japan; ${ }^{3}$ Department of Internal Medicine, Keio University School of Medicine, Tokyo, Japan; ${ }^{4}$ Department of \\ Pathology, Keio University, School of Medicine, Tokyo, Japan; ${ }^{5}$ Department of Laboratory Medicine, Mito Red Cross Hospital, \\ Ibaraki, Japan. \\ Email: ${ }^{*}$ hiroko@a2.keio.jp
}

Received August $7^{\text {th }}, 2013$; revised September $1^{\text {st }}, 2013$; accepted September $8^{\text {th }}, 2013$

Copyright (C) 2013 Tetsuhiro Yoshino et al. This is an open access article distributed under the Creative Commons Attribution License, which permits unrestricted use, distribution, and reproduction in any medium, provided the original work is properly cited.

\begin{abstract}
Invasive aspergillosis is a life-threatening opportunistic infection that occurs most often in immunocompromised patients, especially those with either hematological malignancies treated with chemotherapy or in organ transplant recipients. Here, we report 2 cases of disseminated invasive aspergillosis with myocarditis proven pathologically by autopsy. These 2 cases occurred in immunocompromised patients: the first patient received chemotherapy for acute myeloid leukemia with myelodysplasia-related changes (AML-MRC); the second patient received methotrexate therapy for rheumatoid arthritis (RA). Invasive aspergillosis with myocarditis has high morbidity and mortality rates, therefore it is necessary to make prompt diagnosis and to start intensive treatments quickly.
\end{abstract}

Keywords: Invasive Aspergillosis; Myocarditis; Acute Myeloid Leukemia; Rheumatoid Arthritis

\section{Introduction}

Aspergillus species have the ability to cause aggressive invasive infections in a variety of major organs, including the lungs, heart, central nervous system, and gastrointestinal tract. These organisms most commonly affect a variety of immunocom promised patients with hematological malignancies, especially those who had acute leukemia under receiving chemotherapy, or organ-transplant patients [1]. Here, we present 2 cases of disseminated invasive aspergillosis with myocarditis in immunocom promised patients who received either chemotherapy for acute myeloid leukemia (AML) or immunosuppressive therapy for rheumatoid arthritis (RA).

\section{Case Report 1}

A 76-year-old man presented with asymptomatic anemia in August 2009. The laboratory findings at the initial presentation were as follows: white blood cell (WBC) count, $2.31 \times 10^{3} / \mathrm{mm}^{-3}$ (23\% neutrophils, 54\% lymphocytes, $10 \%$ monocytes, $11 \%$ basophils, and $2 \%$ eosinophils); hemoglobin (Hgb) level, $9.1 \mathrm{~g} / \mathrm{dL}$; platelet (Plt) count,

${ }^{*}$ Corresponding author.
$11.2 \times 10^{4} / \mathrm{mm}^{-3}$. Bone marrow aspiration showed a normocellular bone marrow consisting of less than $5 \%$ myeloblasts, accompanied by dysplasia of erythroblasts and megakaryocytes. Cytogenetic analysis revealed abnormalities with 46XY, del(7) (q31q34) in 5 of 20 cells. The patient was diagnosed with myelodysplastic syndrome with refractory cytopenia with multilineage dysplasia (MDS; RCMD). During his clinical course, anemia and thrombocytopenia had gradually progressed. In August 2011 , leukocytosis with the presence of $49.5 \%$ immature monocytes was indicated. Bone marrow aspiration showed widespread infiltration of CD13+/CD33+/HLA-DR+ immature monocytes which was weakly stained with both peroxidase and non-specific esterase. Cytogenetic abnormalities, including 46XY, $\operatorname{del}(7)$ (q31q34) (in 20 of 20 cells), $+8,+15$ (in 5 of 20 cells), were also observed. Based on these results, the diagnosis of acute myeloid leukemia with myelodysplasia-related changes (AMLMRC) was made. Immediately, low-dose cytarabine chemotherapy (Ara-C: $20 \mathrm{mg} \cdot \mathrm{m}^{-2} /$ day, 14 days) was initiated. However, shortly after initiating Ara-C, the patient developed high fever which was considered to be due to either novel infections or adverse effects of Ara-C. 
Anti-fungal therapy with liposomal amphotericin B (AMPH-B), as well as anti-micobacterial therapy was initiated. However, the patient developed severe neutropenia with a WBC count of $0.2 \times 10^{3} / \mathrm{mm}^{-3}$ and he went into shock. An electrocardiogram (EKG) showed both an elevated ST-T segment in the broad leads and decreased wall motion, with a low echo region at the apex. The patient's clinical course strongly indicated a diagnosis of fungal myocarditis, therefore the therapy with methylpredonisoline (m-PSL; $0.5 \mathrm{mg} / \mathrm{kg} /$ day, 3 days) was initiated. Micafungin (MCFG) was also initiated in addition to liposomal AMPH-B, but no response was obtained and the patient died. The autopsy findings showed disseminated invasive aspergillosis involving the lungs, heart, liver and gastrointestinal tracts (Figures 1(a)-(f)). Leukemia cells were not detected in the extremely hypocellular bone marrow, indicating that leukemia progression had been denied.

\section{Case Report 2}

An 81-year-old woman was admitted to our hospital in October 2011 because of a worsening cough, sore throat, hemorrhagic stomatitis and melena. Progressive pancytopenia; WBC count, $0.23 \times 10^{3} / \mathrm{mm}^{-3}$ with $20 \%$ granulocytes and $80 \%$ lymphocytes was also present. She had been diagnosed with Rhematoid artiritis (RA) at another clinic at age 76 and had started undergoing hemodialysis
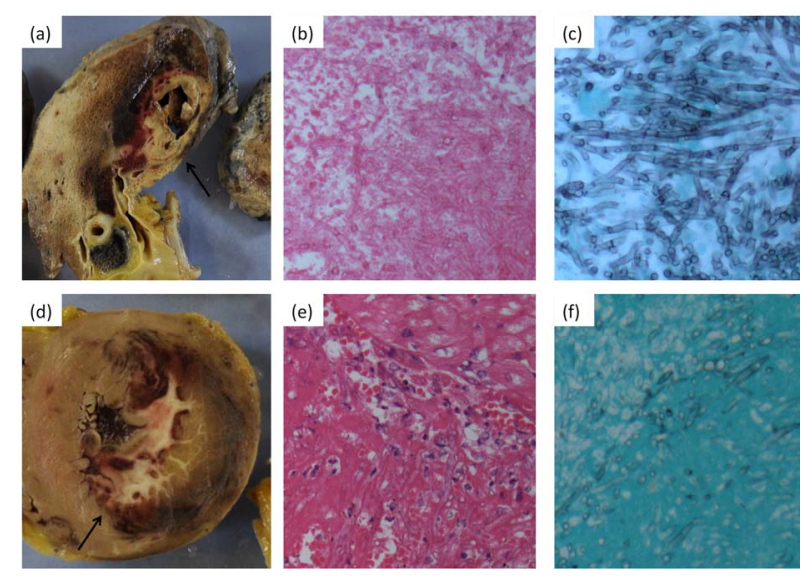

Figure 1. Autopsy results for Case 1, with evidence of invasive fungal infection (hyphae of Aspergillus). (a) Macroscopic image of the right upper lobe of the lung showing cavity lesions with hemorrhagic necrosis (arrow); (b) Diffuse hemorrhagic necrosis was observed in the lung lesion (hematoxylin and eosin [HE] stain; original magnification, $\times 100$ ); (c) Grocott stain for Y-angle branching hyphae demonstrating massive lung tissue invasion (original magnification, $\times 400$ ); (d) Macroscopic image of the heart, showing hemorrhagic necrosis in the left ventricle (arrow); (e) Inflammatory infiltration in the necrotic myocardium (HE stain; original magnification, $\times 100$ ); (f) Grocott staining for the presence of fungi in the myocardium (Grocott stain; original magnification, $\times 400$ ). for chronic renal failure in May 2011. Ten days before her admission, a single dose of methotrexate (MTX; 2 $\mathrm{mg}$ ) was administered to control the disease activity of RA. On her admission, she had high fever and Escherichia coli was detected in the blood culture, therefore anti-micobacterial therapy was immediately initiated. She was also treated with granulocyte-colony stimulating factor (G-CSF: $250 \mu \mathrm{g} /$ day) intraveneously. During her hospitalization, the pancytopenia gradually improved. However, the patient suddenly lost her consciousness and went into shock. Chest imaging did not show any abnormal signs. Computed tomography scan of the head revealed that she had multiple cerebral infarctions. Since Candida tropicalis and Bacteroides fragilis were simultaneously detected in the blood culture, the therapy with MCFG and metronidazole was started. However, despite these supportive treatments, her respiratory symptoms worsened and she died. An EKG showed no definite change. The autopsy findings showed disseminated invasive aspergillosis affecting the lungs, heart, spleen, and kidneys (Figures 2(a)-(f)). The bone marrow showed almost normal cellularity. Although the cause of the cerebral infarctions could have been embolization caused by Aspergillus species, the patient's family did not consent to opening her skull for autopsy.

\section{Discussion}

Invasive aspergillosis is a serious opportunistic infection that occurs in a variety of immunocompromised patients,
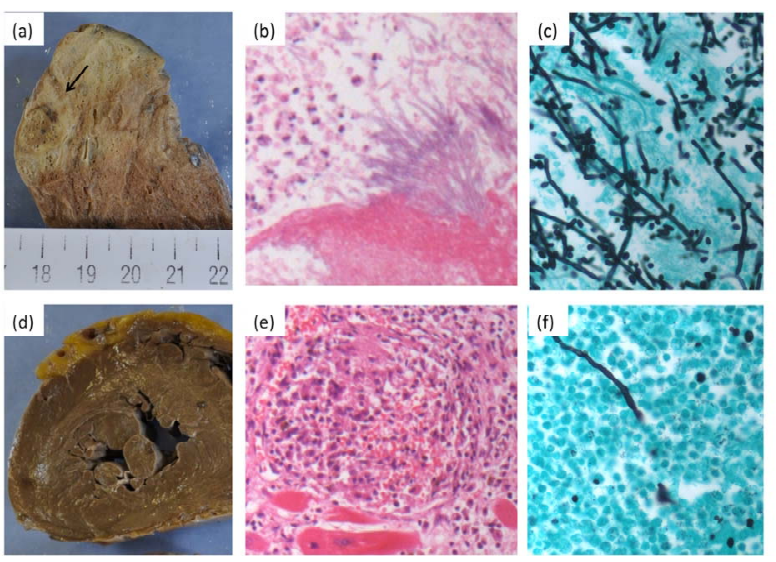

Figure 2. Autopsy results for Case 2, with evidence of invasive fungal infection (hyphae of Aspergillus); (a) Macroscopic image of the left upper lobe of the lung, showing a 10-mm nodule (arrow); (b) Fungal hyphae are seen proliferating radially from the lung wall (HE staining; original magnification, $\times 100$ ); (c) Grocott staining for Y-angle branching fungal hyphae in the lung tissue (original magnification; $\times 400$ ); (d) Macroscopic image of the heart; (e) Abscess lesion observed in the myocardium (HE stain; original magnification, $\times 100$ ); (f) Grocott staining for the presence of fungal hyphae in the myocardium (original magnification, $\times 400)$. 
such as those receiving chemotherapy for hematological malignancies or organ-transplant patients and it has high morbidity and mortality rates. Its incidence has been estimated at around $10 \%-14 \%$, and the mortality rates may be as high as $27 \%-60 \%$ in spite of antifungal therapy $[2,3]$. In order to reduce mortality rates, to make a diagnosis quickly and to initiate intensive treatments are necessary. However, premortem diagnosis of invasive aspergillosis is difficult, because cultures were unreliable and to assess to the sites was limited [2,3].

Autopsy results in Japan have shown that the prevalence of visceral mycoses markedly increased from 3.7\% in 1993 to a peak of $4.6 \%$ in 2009. Aspergillus became the predominant causative pathogen and the rate of aspergillosis exceeded that of candidiasis in 1994; it has continued to increase conspicuously and was even higher in $2001(46.0 \%)[4,5]$. On the other hand, since 2001, there has been a slight decrease in the frequency of aspergillosis because of the development of novel, effective antifungal agents and advancements in non-invasive diagnostic tools. In addition, an increasing number of cases with collagen diseases as the underlying cause of visceral mycosis and a decreasing number of cases with leukemia as the underlying disorders were noted. However, overall, invasive aspergillosis are still one of the most serious infections not only in patients with malignant diseases but also in those with recieving steroids or immunosuppressive therapy for a variety of diseases $[4,5]$.

The lung is the most common primary site of invasive aspergillosis with secondary hematogenous dissemination. Once Aspergillus forms abscesses in the lung, it can invade blood vessels and disseminate to the entire body. Cases of cardiac invasive fungal infection is difficult to prove premortem and has an especially high mortality rate. In the case of Aspergillus endocarditis, the mortality rate reaches $96 \%$ if the patient is treated by medication alone, and $68 \%$ even if surgical resection is performed [6-9]. It is difficult to make a diagnosis of aspergillus myocarditis, because it requires histopathological evidence of characteristic fungal hyphae or positive culture results, yielding aspergillus species from myocardial specimens. Our patients discussed here showed disseminated invasive aspergillosis with myocarditis that was proven by autopsy. In Case 1, hemorrhagic necrosis was shown in the left ventricle and the presence of fungal hyphae in the myocardium was demonstrated. In Case 2, Abscess lesion observed in the myocardium and fungal hyphae in the myocardium was also detected. Both cases had several common risk factors for developing disseminated invasive aspergillosis. These factors include the underlying disease, long-term immunosuppressive therapies including steroids, prolonged neutropenia or neutrophil dysfunction and the use of broad-spectrum antibiotics which affect mucosal barriers [2,3]. Leukemia and MDS have been the major underlying diseases to develop invasive aspergillosis, followed by solid cancers and bacterial infections and only a few reports have been published in which invasive aspergillosis with myocarditis was proven pathologically $[4,6-8,10]$. In Case 2 , pancytopenia due to severe bone marrow suppression occurred after the patient received MTX. It was considered to be an adverse effect of MTX due to renal insufficiency and it may became the risk factor to develop invasive aspergillosis with myocarditis in Case 2. In most reported cases, it is difficult to determine what to extent the myocarditis contributed to the patient' death. The clinical course of aspergillus myocarditis is extremely short and results in fatality, so EKG changes consistent with severe symptoms was not necessarily revealed. Williams reported only 6 cases of 37 cases of secondary aspergillosis in which the myocardium was involved had electrocardiographic changes consistent with ischemia of myocardial infarction [11]. In our case, Case 1 showed positive electrocardiographic and echographic changes, correlating with ischemia. We believe that our cases certainly died of asperugillus myocarditis and subsequent heart failure, by its extremely quick clinical course, laboratory and necropsy findings and the absence of other alternative etiologocal findings.

\section{Conclusion}

In conclusion, we reported 2 cases of disseminated invasive aspergillosis with myocarditis, which were all confirmed by autopsy. These 2 cases involved immunocompromised patients receiving chemotherapy or immunosuppressive therapies. Invasive aspergillosis, especially in cardiac infection including myocarditis, has high morbidity and mortality rates. It is therefore necessary to provide early diagnosis and intensive treatments, including novel antifungal therapies.

\section{REFERENCES}

[1] T. J. Walsh, E. J. Anaissie, D. W. Denning, et al., "Treatment of Aspergillosis: Clinical Practice Guidelines of the Infectious Diseases Society of America," Clinical Infectious Diseases, Vol. 46, No. 3, 2008, pp. 327-360. http://dx.doi.org/10.1086/525258

[2] P. Eggimann, J. C. Chevrolet, M. Starobinski, et al., "Primary Invasive Aspergillosis of the Digestive Tract: Report of Two Cases and Review of the Literature," Infection, Vol. 34, No. 6, 2006, pp. 333-338. http://dx.doi.org/10.1007/s15010-006-5660-0

[3] L. Pagano, M. Caira, A. Candoni, et al., "Invasive Aspergillosis in Patients with Acute Myeloid Leukemia: A SEIFEM-2008 Registry Study," Haematologica, Vol. 95, No. 4, 2010, pp. 644-650.

http://dx.doi.org/10.3324/haematol.2009.012054 
[4] H. Kume, T. Yamazaki, T. Togano, et al., "Epidemiology of Visceral Mycoses in Autopsy Cases in Japan: Comparison of the Data from 1989, 1993, 1997, 2001, 2005 and 2007 in Annual of Pathological Autopsy Cases in Japan," Medical Mycology Journal, Vol. 52, No. 2, 2011, pp. 117-127. http://dx.doi.org/10.3314/jimm.52.117

[5] Y. Suzuki, H. Kume, T. Togano, Y. Kanoh and H. Ohto, "Epidemiology of Visceral Mycoses in Autopsy Cases in Japan: The Data from 1989 to 2009 in the Annual of Pathological Autopsy Cases in Japan," Medical Mycology, Vol. 51, No. 5, 2013, pp. 522-526. http://dx.doi.org/10.3109/13693786.2012.755574

[6] H. Kume, T. Yamazaki, M. Abe, H. Tanuma, M. Okudaira and I. Okayasu, "Increase in Aspergillosis and Severe Mycotic Infection in Patients with Leukemia and MDS: Comparison of the Data from the Annual of the Pathological Autopsy Cases in Japan in 1989, 1993 and 1997," Pathology International, Vol. 53, No. 11, 2003, pp. 744-750.

http://dx.doi.org/10.1046/j.1440-1827.2003.01548.x

[7] P. Nenoff, C. Kliem, M. Mittag, L. C. Horn, D. Niederwieser and U. F. Haustein, "Secondary Cutaneous Aspergillosis Due to Aspergillus Flavus in an Acute Myeloid

\section{Abbreviations}

AML-MRC: acute myeloid leukemia with myelodysplasia-related changes;

RA: rheumatoid arthritis;

WBC: white blood cell;

Hgb: hemoglobin;

Plt: platelet;

MDS-RCMD: myelodysplastic syndrome with refractory
Leukaemia Patient Following Stem Cell Transplantation," European Journal of Dermatology, Vol. 12, No. 1, 2002, pp. 93-98.

[8] T. Yamazaki, H. Kume, S. Murase, E. Yamashita and M. Arisawa, "Epidemiology of Visceral Mycoses: Analysis of Data in Annual of the Pathological Autopsy Cases in Japan," Journal of Clinical Microbiology, Vol. 37, No. 6, 1999, pp. 1732-1738.

[9] A. S. Kalokhe, N. Rouphael, M. F. El Chami, K. A. Workowski, G. Ganesh and J. T. Jacob, "Aspergillus Endocarditis: A Review of the Literature," International Journal of Infectious Diseases, Vol. 14, No. 12, 2010, pp. e1040-e1047. http://dx.doi.org/10.1016/j.ijid.2010.08.005

[10] P. C. Iwen, M. E. Rupp, M. R. Bishop, et al., "Disseminated Aspergillosis Caused by Aspergillus Ustus in a Patient Following Allogeneic Peripheral Stem Cell Transplantation," Journal of Clinical Microbiology, Vol. 36, No. 12, 1998, pp. 3713-3717.

[11] A. H. Williams, "Aspergillus Myocarditis," American Journal of Clinical Pathology, Vol. 61, No. 2, 1974, pp. 147-256.

cytopenia with multilineage dysplasia;

Ara-C: cytarabine arabinoside;

AMPH-B: amphotericin B;

EKG: electrocardiogram;

MCFG: micafungin;

MTX: methotrexate;

G-CSF: granulocyte-colony stimulating factor. 